\title{
TSIRI PUMA, HÍBRIDO DE MAÍZ PARA VALLES ALTOS CON ESQUEMA DE ANDROESTERILIDAD PARA PRODUCCIÓN DE SEMILLAS
}

\section{TSIRI PUMA, A MAIZE HYBRID FOR THE MEXICAN HIGH VALLEYS WITH AN ANDROSTERILE SCHEME FOR SEED PRODUCTION}

\author{
Margarita Tadeo-Robledoํ, Alejandro Espinosa-Calderón²*, J. Jesús García-Zavala ${ }^{3}$, \\ Ricardo Lobato-Ortiz ${ }^{3}$, Noel O. Gómez-Montiel ${ }^{4}$, Mauro Sierra-Macías ${ }^{5}$, Roberto Valdivia-Bernal ${ }^{6}$, \\ Benjamín Zamudio-González², Beatriz Martínez-Yañez' ${ }^{1}$ Consuelo López-López' ${ }^{1}$ Karina Y. Mora-García', \\ Enrique I. Canales-Islas ${ }^{1}$, Alma L. Cárdenas-Marcelo', Job Zaragoza-Esparza' y Hugo J. Alcántar- Lugo ${ }^{1}$
}

\begin{abstract}
'Ingeniería Agrícola, Facultad de Estudios Superiores Cuautitlán, Universidad Nacional Autónoma de México (FESC-UNAM). Carretera CuautitlánTeoloyucán, Km 2.5. 54714, Cuautitlán Izcalli, Estado de México. ${ }^{2}$ Campo Experimental Valle de México, Instituto Nacional de Investigaciones Forestales, Agrícolas y Pecuarias (INIFAP). Km 13.5 Carr. Los Reyes-Texcoco. 56250, Coatlinchán, Texcoco, Estado de México. ${ }^{3}$ Postgrado en Recursos Genéticos y Productividad-Genética, Colegio de Postgraduados. Km. 36.5 Carr. México-Texcoco. 56230, Montecillo, Texcoco, Estado de México. ${ }^{4}$ Campo Experimental Iguala, INIFAP. Km. 2.5 Carr.Iguala-Tuxpan. 40000, Col. Centro Tuxpan, Iguala de la Independencia Guerrero. ${ }^{5}$ Campo Experi-

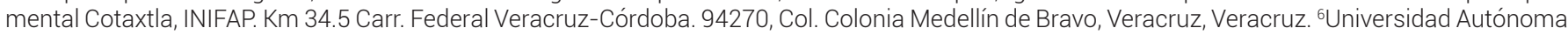
Nayarit. Ciudad de la Cultura Amado Nervo S/N. 63155, Los Frenos, Tepic, Nayarit.
\end{abstract}

*Autor de correspondencia (espinoale@yahoo.com.mx.)

En Valles Altos de México (de 2200 a 2600 msnm), el área sembrada con maíz (Zea mays L.) en condiciones de riego, humedad residual o precipitaciones pluviales favorables, es aproximadamente de 700 mil hectáreas (Turrent, 1994). De esta superficie, se estima que en al menos 300,000 hectáreas se podrían sembrar híbridos de alto rendimiento. El rendimiento promedio que se obtiene en estas condiciones es 3.5 t ha $^{-1}$ de grano de maíz, aunque puede incrementarse a $6.0 \mathrm{t} \mathrm{ha}^{-1}$ si se siembran semillas mejoradas y se aplican las tecnologías de producción desarrolladas por el Instituto Nacional de Investigaciones Forestales, Agrícolas y Pecuarias (INIFAP).

En los lotes de producción comercial de semilla híbrida de maíz, al progenitor femenino generalmente se le elimina la espiga (acción llamada 'desespigar') para mantener la calidad genética de la semilla; esta operación requiere de 24 a 50 jornales ha-1 según su uniformidad, presencia de tallos secundarios y facilidad para retirar la espiga (Martínez-Lázaro et al., 2005; Tadeo et al., 2003). Una alternativa para eliminar el desespigamiento es el uso de progenitores femeninos androestériles (Espinosa et al., 2012; Martínez et al., 2005).

Desde 1992, investigadores del INIFAP y de la Universidad Nacional Autónoma de México (UNAM) iniciaron la incorporación de la esterilidad masculina a los progenitores de algunos híbridos de maíz (Tadeo et al., 2003; Tadeo et al., 2010; Tadeo-Robledo et al., 2014a). Como resultado de este proceso, en la UNAM se generó el híbrido de maíz denominado Tsiri Puma, que se registró de forma definitiva en el Catálogo Nacional de Variedades Vegetales (CNVV) con el número MAZ-1571-290514, y actualmente está en trámite el Título de Obtentor. En este escrito se presenta información del híbrido Tsiri Puma, sus características agronómicas, rendimiento y otras características que lo ubican como alternativa de uso para los productores de maíz de Valles Altos (Tadeo et al., 2010; Tadeo-Robledo et al., 2014a; Tadeo-Robledo et al., 2014b).

Tsiri Puma es un híbrido trilineal, de grano blanco y textura semidentada, con adaptación favorable a Valles Altos (de 2200 a 2600 msnm); sin embargo, prospera bien desde 1900 a 2600 msnm. En fechas de siembra del 15 de mayo al 20 de abril, con densidad de población de 65,000 plantas $\mathrm{ha}^{-1}$, es de madurez intermedia ya que su floración masculina ocurre a los $84 \mathrm{~d}$ y la femenina a los $86 \mathrm{~d}$. En altitud de 2250 m es ligeramente más tardío que el híbrido comercial H-48. Llega a madurez fisiológica a los 161 d, después de lo cual se puede cortar y 'amogotar' (secado en campo), para luego efectuar la pizca manual; de ser el caso, la cosecha mecánica puede efectuarse a los $175 \mathrm{~d}$.

Como se ilustra en la Figura 1, la altura de planta varía de 255 a $270 \mathrm{~cm}$ y la de mazorca de 127 a $140 \mathrm{~cm}$; la longitud de mazorca es de $16.5 \mathrm{~cm}$, con 16 hileras y 32 granos por hilera; y su relación grano/mazorca es de $86 \%$. Es resistente al acame de raíz y tallo, y a las enfermedades fungosas de planta y mazorca causadas por los géneros Puccinia y Ustilago. Su rendimiento promedio de grano, a través de varios años (2008 a 2012) y localidades en el Estado de México (Cuautitlán, Ixtlahuaca, Texcoco, Temascalcingo, Atlacomulco), varió de 9.5 a $12.5 \mathrm{t} \mathrm{ha}^{-1}$ con un rendimiento medio de $8.5 \mathrm{t} \mathrm{ha}^{-1}$, valor superior en $20 \%$ al H-48. 




Figura 1. Planta (a), elote (b), mazorca y grano (c) del híbrido de maíz Tsiri Puma.

Tsiri Puma expresa buena productividad en ensilado, ya que su rendimiento de forraje es de 83,369 $\mathrm{kg} \mathrm{ha}^{-1}$, con materia seca de $24,623 \mathrm{~kg} \mathrm{ha}^{-1}$ y porcentaje de digestibilidad $72.47 \%$, por lo que se recomienda para doble propósito. El rendimiento de masa es superior a lo requerido por los industriales de la masa-tortilla (2.05 kg de masa / kg de maíz procesado), con peso hectolítrico de $79.4 \mathrm{~kg} \mathrm{hL}^{-1}$ e índice de flotación de $16 \%$.

En el híbrido Tsiri Puma participan las líneas IA424F, IA424AEC, IA249 e IA449. La línea IA424F se obtuvo de germoplasma del Centro Internacional de Mejoramiento de Maíz y Trigo (CIMMYT), a partir de selecciones en autofecundaciones y cruzamientos planta a planta y ciclos de avance fraternal, hasta obtener la línea MIU424 que finalmente se denominó línea IA424F. La línea IA424 AEC fue desarrollada en la UNAM al incorporarle a la línea IA424F, la fuente de androesterilidad tipo $C$ de acción génicocitoplásmica, propiedad de la UNAM, con la genealogía CXP3PL 7, que posee estabilidad en la expresión del carácter a través de ambientes.

Esta línea IA424F fue utilizada como progenitor masculino donador de polen para cruzarse con la fuente de androesterilidad, y posteriormente se le efectuaron seis retrocruzas hacia la línea IA424F (mantenedora) para contar con la versión de esterilidad masculina de la línea, por lo que su denominación fue IA424AEC (androestéril), que es la versión isogénica y cuya mantenedora es la propia línea IA424F en su versión fértil. La línea IA249 proviene de la Raza Cónico, derivada y avanzada en la UNAM mediante el método genealógico a partir de la generación F2 de un híbrido obtenido en la propia UNAM. La línea IA449 fue derivada y avanzada en la UNAM también mediante el método genealógico, a partir de la generación F2 de un maíz comercial.

La producción de semilla del híbrido Tsiri Puma es sencilla, ya que la exposición de los estigmas de sus progenitores femeninos, las cruzas simples hembras (IA424AEC x IA249) y (IA424F x IA249), versión androestéril y versión fértil respectivamente, coinciden en la liberación de polen (86 d en ambos casos) con la línea IA449 macho del híbrido trilineal. Ambas cruzas simples muestran alta productividad, ya que rinden de 7.5 a 9.0 t ha $^{-1}$ de semilla comercial, con tamaño mediano y grande, y alta proporción de semilla plana $(70 \%)$.

En un mismo lote de producción del híbrido trilineal se deben sembrar ambas cruzas simples en la proporción $80 \%$ androestéril y $20 \%$ fértil, ya que el macho que poliniza a ambas para formar el híbrido trilineal es el mismo; entonces se establecen relaciones de 6 surcos de cruza simple y 2 surcos de la línea macho en las franjas que 
correspondan de cruzas simples hembras androestériles, hasta completar $80 \%$ de la superficie; después se establecen franjas de la cruza simple fértil hasta completar el 20 $\%$ restante de la superficie. En la cosecha se mezclan mazorcas de hembras androestériles y fértiles para obtener la proporción 80:20 señalada, lo cual disminuye los costos de producción porque únicamente se 'desespigan' las plantas de la cruza simple hembra (20\%), no así los surcos de las fajas con cruza simple androestéril que representan el 80 $\%$ de la superficie.

Lo anterior favorece el control de calidad genética de la semilla híbrida que se obtiene, con una productividad media de 8116 t ha $^{-1}$. La producción de semilla debe hacerse bajo riego, en siembras de abril y mayo, con 65,000 plantas ha-1 en el Valle de México, Valle de Toluca, Atlacomulco, Valle de Puebla, San Martín Texmelucan, y en Huamantla y Apizaco en el Estado de Tlaxcala. La semilla registrada se puede adquirir en la Facultad de Estudios Superiores Cuautitlán (FESC) de la UNAM localizada en Cuautitlán Izcalli, para incrementar semilla certificada con la inspección y calificación del Servicio Nacional de Inspección y Certificación de Semillas (SNICS).

\section{AGRADECIMIENTOS}

Se agradece el apoyo financiero del Programa de Apoyo a Proyectos de Investigación e Innovación Tecnológica a través del proyecto PAPIIT: 201215. DGAPA-UNAM.

\section{BIBLIOGRAFÍA}

Espinosa C. A., M. Tadeo R., J. Virgen V., I. Rojas M., N. Gómez M., M. Sierra M., A. Palafox C., G. Vázquez C., F. Rodríguez M., B. Zamudio G., I. Arteaga E., E. I. Canales I., B. Martínez Y. y R. Valdivia B. (2012) H-51 AE, híbrido de maíz para áreas de humedad residual, buen temporal y riego en Valles Altos del centro de México. Revista Fitotecnia Mexicana 35: 347-349.

Martínez-Lázaro C., L. E. Mendoza-Onofre, S. G. García-Santos, M. C. Mendoza-Castillo y A. Martínez-Garza (2005) Producción de semilla híbrida de maíz con líneas androestériles y androestériles-isogénicas y su respuesta a la fertilización y densidad de población. Revista Fitotecnia Mexicana 28:127-133.

Tadeo R. M., A. Espinosa C., A. M. Solano y R. Martínez M. (2003) Androesterilidad en líneas e híbridos de maíz de Valles Altos de México. Agronomía Mesoamericana 14:15-19.

Tadeo R. M., A. Espinosa C., J. Serrano R., M. Sierra M., F. Caballero H., R. Valdivia B., N. Gómez M., A. Palafox C., F. A. Rodríguez M. y B. Zamudio G (2010) Productividad de diferentes combinaciones de semilla androestéril y fértil en dos híbridos de maíz. Revista Mexicana de Ciencias Agrícolas 1:273-287.

Tadeo-Robledo M., A. Espinosa-Calderón, A. Turrent-Fernández, B. ZamudioGonzález, R. Valdivia-Bernal y P. Andrés-Meza (2014a) Productividad de grano de cuatro híbridos trilineales de maíz en versión androesteril y fértil. Agronomía Mesoamericana 25:45-52.

Tadeo-Robledo M., A. Espinosa-Calderón, A. Turrent-Fernández, B. ZamudioGonzález, M. Sierra-Macías, N. Gómez-Montiel, R. Valdivia-Bernal y J. Virgen-Vargas (2014b) Rendimiento de híbridos androestériles y fértiles de maíz en dos localidades en Valles Altos de México. Revista Mexicana de Ciencias Agrícolas 5: 883-891.

Turrent F. A (1994) Plan de Investigación del Sistema Maíz-Tortilla en la Región Centro. CIRCE, INIFAP, SARH, Publicación Especial No. 12, Chapingo, México. 